\title{
THE USE OF LINEAR SUPERCONDUCTING ELECTRON ACCELERATOR FOR SUBCRITICAL REACTOR DRIVING
}

\author{
I. S. Guk, A. N. Dovbnya, S. G. Kononenko, F. A. Peev, A. S. Tarasenko \\ National Science Centre «Kharkov Institute of Physics and Technology», Kharkov, Ukraine
}

\section{M. van der Wiel, J. I. M. Botman}

Technische Universiteit Eindhoven, Eindhoven, the Netherlands

At NSC KIPT the opportunity of creating the installation with subcritical reactor, driven by the electron accelerator, is examined. For obtaining the maximal stream of neutrons from neutron-producing target at minimal density of energy emission, the electron energy should lay in the range 100-200 MeV, and the size of target should be maximal. The other important requirements are the beam continuity in time and the long-term stability of the accelerator parameters. The variants of using the superconducting linear accelerator on the basis of accelerating structure TESLA as a driver of subcritical reactor are considered. The basic design parameters and characteristics of this installation are submitted.

В ННЦ ХФТИ рассматривается возможность создания установки с подкритическим реактором, управляемым электронным ускорителем. Для получения максимального потока нейтронов из нейтронопроизводящей мишени при минимальной плотности энерговыделения энергия электронов должна лежать в диапазоне 100-200 МэВ, а размер мишени должен быть максимальным. Другим важным требованием является непрерывность пучка во времени и долговременная стабильность параметров ускорителя. Рассмотрены возможности использования сверхпроводящего линейного ускорителя на основе ускоряющей структуры TESLA как драйвера подкритического реактора. Приводятся проектные параметры и характеристики установки.

PACS: 29.20.Ej

\section{INTRODUCTION}

Recently in a number of scientific centres, including NSC KIPT [1], the opportunity of creating the subcritical reactor driven by the electron accelerator is considered. For the prompt starting up the installation mentioned above, it is supposed to use the fuel elements, which are made serially on well fulfilled technology. However, they all are designed to operate in conditions of constant or slowly varying in time thermal and radiation loadings. The use of the pulsed accelerator as the driver means that the fuel elements and the neutron-producing target will be exposed to the action of pulsed neutron stream and thermal emission which more than 104 times surpass the average values of these magnitudes. The data on operation in these conditions of the stock-produced fuel elements are absent. Taking into account this fact, the requirement of driver beam continuity is natural. The use of superconducting accelerating structures working in a continuous mode allows one to solve this problem.

In the present report, opportunities of using electron beam of accelerating complex SALO [2,3] for operation with neutron-producing target of installation with subcritical assembly are observed. 


\section{THE SUPERCONDUCTING DRIVER ON THE BASIS OF SALO FACILITY}

For operation as the driver of subcritical assembly, SALO facility $[2,3]$ will be equipped with the high-current injector [2,3], which provides the following beam parameters: the rated energy $9.5 \mathrm{MeV}$, the average current $1 \mathrm{~mA}$. Injector is a photogun with superconducting accelerating system on the basis of accelerating structure TESLA. The operating mode is continuous, the maximal frequency is $13 \mathrm{MHz}$, it is caused by the laser used.

For SALO project realization the superconducting accelerating structure TESLA, which was designed at DESY [4], was picked. The adjusted technology of mass production of highfrequency sections allows obtaining the accelerating gradient up to $35 \mathrm{MV} / \mathrm{m}$ in continuous mode.

The sections characteristics are stable during a long time and do not reduce parameters under condition of observance of technology requirements on operation.

Now some types of cryogenic module for different number of sections are developed and made. We had chosen the module with two sections which are brought out by the small lots by the ACCEL firm [5]. Such a module allows obtaining the energy gain $20 \mathrm{MeV}$ with an average current of $1 \mathrm{~mA}$. In the linear accelerator of SALO complex it is supposed to use six such modules. Thus, the beam energy on the accelerator output will be about $130 \mathrm{MeV}$, which corresponds to the requirements on the driver of installation [1].

The experimental halls in which it is planned to place the SALO installation and variants of output beam lines N1, N2, N3, and N4 to subcritical assembly are shown in Fig. 1. The most attractive is variant $\mathrm{N} 4$ as it allows one to implement the design of installation in the shortest terms and to exclude completely capital construction. The subcritical assembly can be disposed in the existing hall of SP-103, which has a powerful radiation protection. However, this variant expels an opportunity of works on nuclear physics with electron energy up to $730 \mathrm{MeV}$. The other three variants will demand construction of building for subcritical assembly.

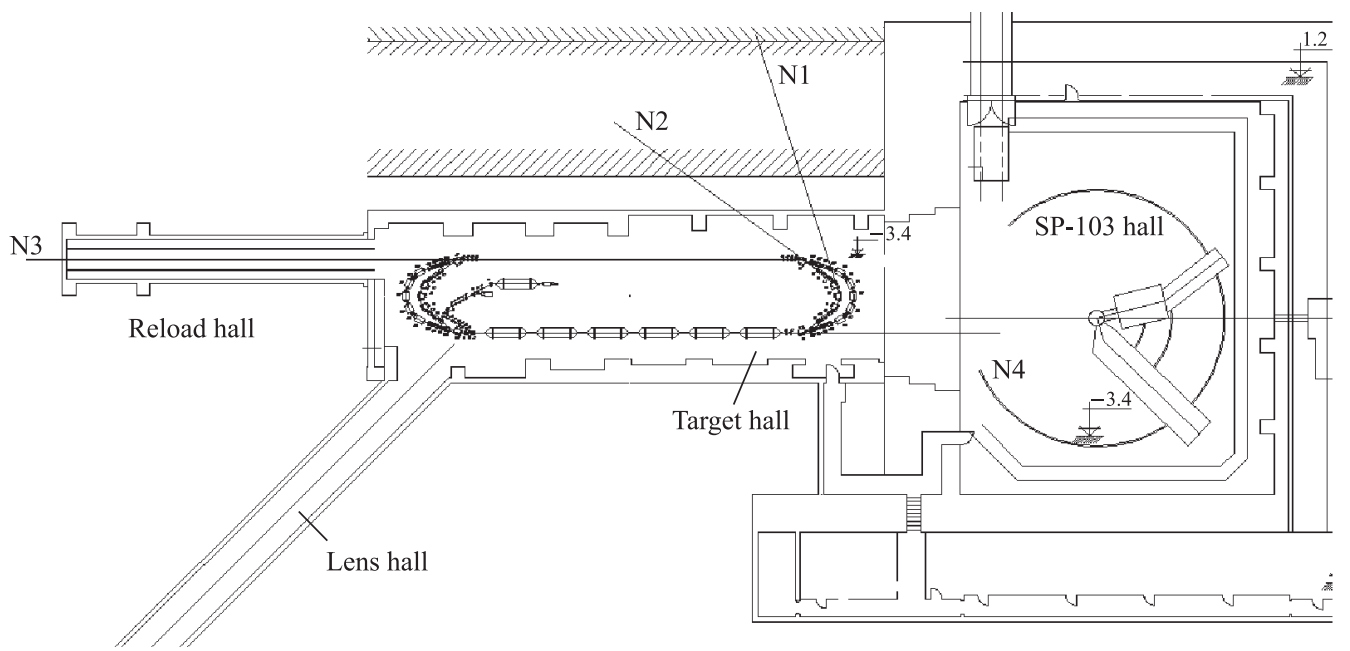

Fig. 1. 


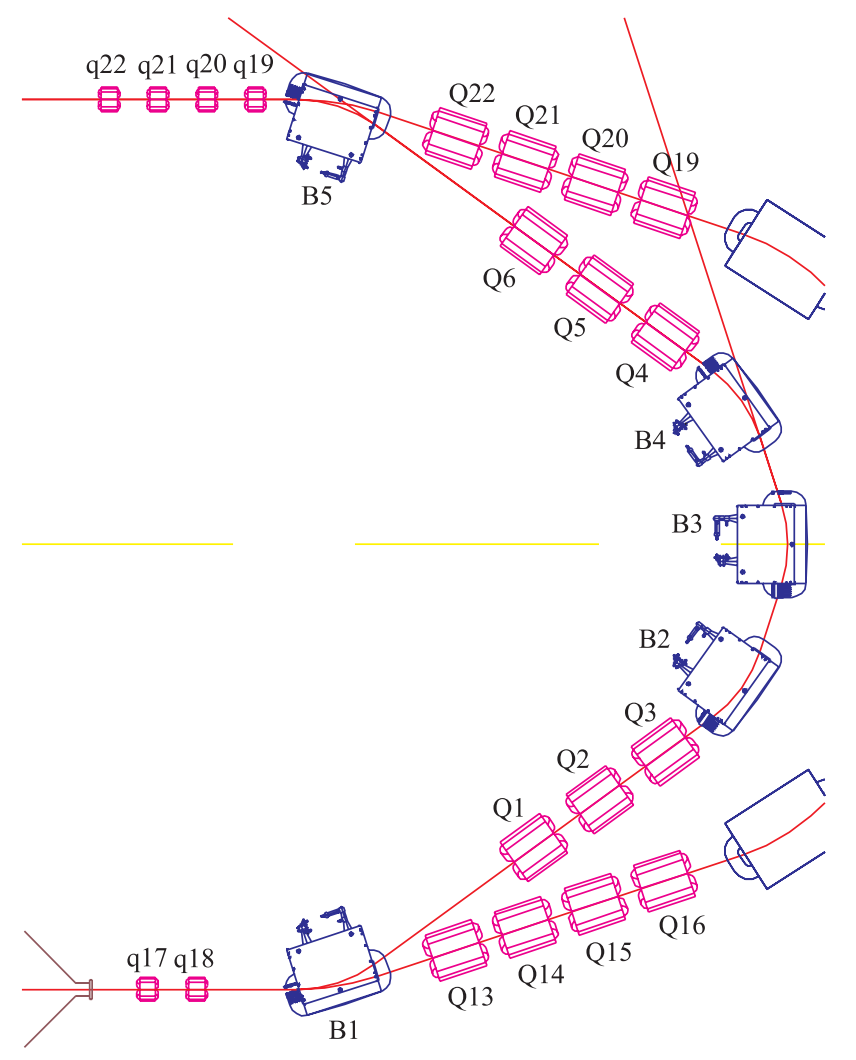

Fig. 2.

For the beam transportation on the target these beam lines will use magnetic devices of the first arch [6] (see Fig. 2). The magnets B1, B2, B3 turn the beam through $108^{\circ}$ and send it into channel N1. Magnets B4 and B5 are used in beam lines N2 and N3.

In each beam line one can allocate two sites: 1) the beam line for beam transporting from recirculator output up to the input of the first vertical magnet BV1 and 2) the beam line of beam rotation in vertical plane and its transporting to neutron-producing target. The last site is the same for all variants of channels. Because all beam lines have no basic differences, it is enough to consider one of them, for example, beam line N1.

The quadrupole lenses Q1-Q3, which are located between magnets B1 and B2, provide the achromaticity of beam line up to the magnet BV1 input. Two quadrupole triplets N1Q1N1Q3 and N1Q4-N1Q6 (see Figs. 3 and 4; on $X$ axis the distance from injector is specified) provide the beam transport from magnet B3 to magnet BV1 over distance of $\sim 58 \mathrm{~m}$ with the following parameters: the maximal horizontal size is $1.7 \mathrm{~cm}$; the maximal vertical size is $0.25 \mathrm{~cm}$. In Fig. 3 are shown vertical and horizontal beam envelopes for this beam line.

The beam rotation through $90^{\circ}$ in a vertical plane is carried out by two $45^{\circ}$ magnets BV1 and BV2 (see Fig. 4).

In this case the beam cross section at the first magnet input is minimal and close to round and it provides the minimal vertical magnet aperture. After this the electron beam with power 


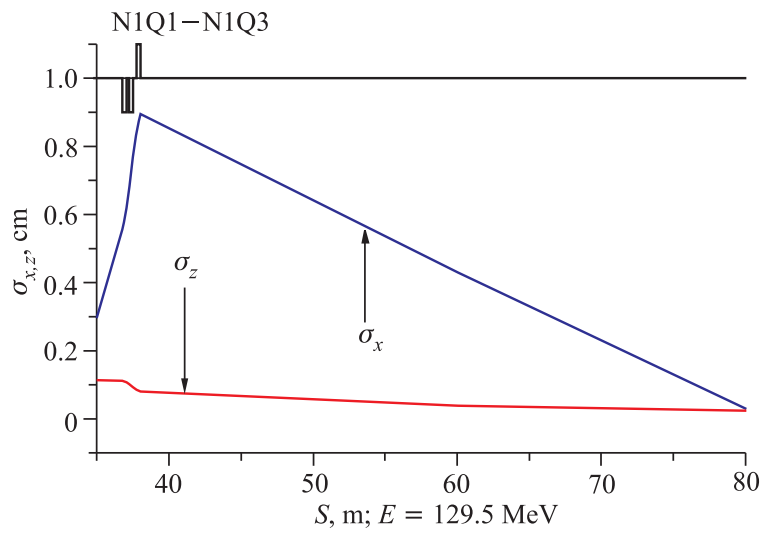

Fig. 3.

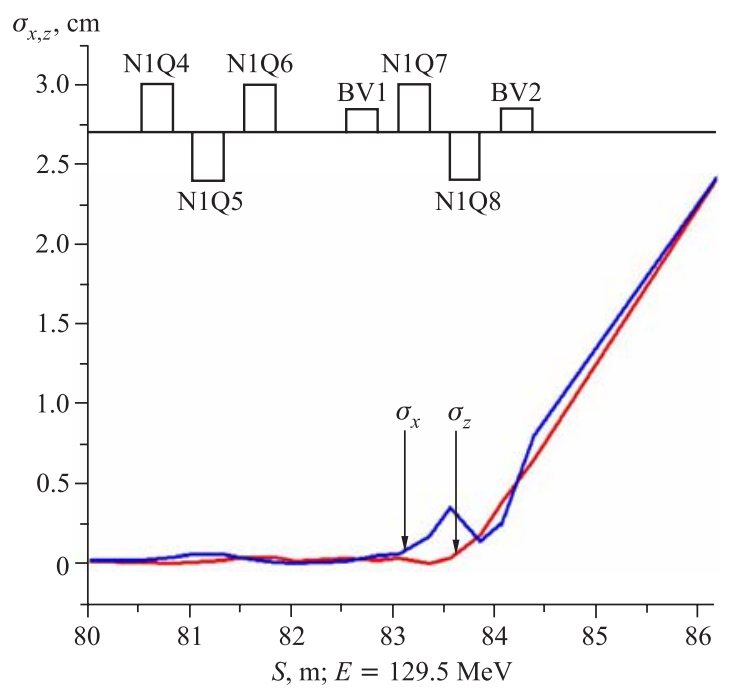

Fig. 4.

$\sim 130 \mathrm{~kW}$ is transported to neutron-producing target, which is located at a distance of $1.9 \mathrm{~m}$ from the edge of magnet BV2 (the distance is defined by the sizes of tank in which subcritical assembly is supposed to be installed). The beam sizes on target should be such that do not allow the target overheating and simultaneously minimize the beam losses on the beam-line walls. At the target diameter $6 \mathrm{~cm}$, the beam diameter on the target should be $\sim 5 \mathrm{~cm}$. In this case the beam losses on the walls of the vacuum chamber will not exceed $1 \%$.

Calculations have shown that in case of providing the necessary beam sizes on the target with the help only of triplets N1Q1-N1Q6 the beam size in magnet BV1 equals $\sim 4 \mathrm{~cm}$ and this demands the vertical aperture of magnets not less than $5 \mathrm{~cm}$. The use in BV-magnets the edge focusing allows one to reduce the vertical aperture; however, in this case the opportunity of beam size adjustment on the target will be lost. In this connection, it is more expedient to adjust the beam sizes on the target with the help of lenses N1Q7 and N1Q8, which are 
located between magnets BV1 and BV2. In this case, the interval behind magnet BV2 is not achromatic. When $\Delta E / E \approx 10^{-4}$, as it is in our case, the energy spread contribution to the beam sizes on the target does not exceed $\sim 1 \mathrm{~mm}$. In Fig. 4 the beam envelope for the beam line from the lens N1Q4 up to neutron-producing target is shown. The given curves and magneto-optical structure appropriate to them are typical for all presumable beam lines.

The research pursued into influence of magnetic elements power supply stability has shown that if the current fluctuations are in limits $\Delta I / I \approx 10^{-4}$, the beam position on the target varies no more than $5 \mathrm{~mm}$.

From the above-mentioned data it is evident that the beam cross sizes in the injection beam line, the transporting beam line and the beam formation beam line do not exceed several millimeters. The vertical aperture of the vacuum chamber is determined by the magnet gap size which amounts to $\sim 22 \mathrm{~mm}$; the horizontal vacuum chamber aperture will be not less than $44 \mathrm{~mm}$. Thus, the beam losses in these beam lines will be much less than $0.1 \%$. The minimal aperture in accelerating structure is $70 \mathrm{~mm}$; therefore, the beam losses in it are still smaller. The beam lines will have the aperture not less than $44 \mathrm{~mm}$; therefore, the beam losses there will be less than $1 \%$. The greatest beam losses on the chamber walls will occur on beam line before the target.

To obtain the minimal beam density on the target, the beam sizes should be maximally close to the target size. In the case of Gauss distribution particles in the beam, the part of it will get on the walls of the beam line near the target. However, it will not give the essential contribution to the beam line material activation, because this part of the beam line will already be in the radiation field of the central zone of subcritical assembly. To diminish the influence of possible beam losses on activation of beam line equipment, it is necessary to choose aluminum as structural material in which irradiation does not form the long-living isotopes.

\section{CONCLUSIONS}

The considered linear electron accelerator with superconducting accelerating structures allows obtaining quasi-continuous electron beam with energy 100-130 MeV and average beam power on the target of 100-130 kW. Together with neutron-producing target from the impoverished uranium, such an installation allows one to obtain the effective driver for subcritical assembly with a full stream $5 \cdot 10^{14}$ photoneutrons/s [7]. The proposed beam transport system provides beam transfer from the output of accelerating structure up to neutron-producing targets with required parameters at the total level of beam losses no more than $1 \%$.

\section{REFERENCES}

1. Proc. of the Ukraine-USA Meeting «Accelerator Driven Subcritical Assembly Facility», Kharkiv, Ukraine, Feb. 24-25, 2005. Kharkiv: NSC KIPT, 2005. 246 p.

2. Arkatov Yu. M. et al. SALO Project. Kharkiv: NSC KIPT, 2005. 104 p.

3. Guk I.S. et al. Accelerating Complex SALO // Proc. of RuPAC, Novosibirsk, Russia, 2006. P. 82-84; http://accelconf.web.cern.ch/AccelConf/r06/PAPERS/TUFO06.PDF 
4. Lilje L. High-Gradient Superconducting Radiofrequency Cavities for Particle Acceleration // Proc. of EPAC, Edinburgh, 2006. P. 2752-2754.

5. Pekeler M., Vogel H., vom Stein P. Industrial Production of Superconducting $1.3 \mathrm{GHz}$ Accelerator Modules and Components for FEL Application // Proc. of the 2004 FEL Conf. P. 379-380.

6. Guk I. S. et al. Isochronous Magneto-Optical Structure of Recirculator SALO // Proc. of EPAC, Edinburgh, 2006. P. 2035-2037.

7. Bratchenko M. I. et al. Superconducting Driver for Subcritical Assembly // Proc. of the Intern. Conf. «Current Problems in Nuclear Physics and Atomic Energy», May 29 - June 3, 2006. Kiev, 2007. P. 622-632. 